\title{
Aerodynamic analysis of railway wagon on drag coefficient
}

\author{
M. Bhagya Lakshmi Nageswari ${ }^{1 *}$, Dr.U.S.Jyothi ${ }^{2}$ \\ ${ }^{1 *}$ PG Student, Mechanical Engineering Department, GRIET, Hyderabad, India \\ ${ }^{2}$ Professor, Mechanical Engineering Department, GRIET, Hyderabad, India
}

\begin{abstract}
Aerodynamics play a major role in transport. When speed increases aerodynamic factors come into existence. By altering the design in an aerodynamic way, it can lead to better efficiency. This paper presents the effect of aerodynamic parameters on the wagon. The analysis was carried out on the existing and modified geometries using ANSYS Fluent 18.1softwareconsidered in the static ground conditions with the wind flowing at zero yaw angles. It was found that the coefficient of drag $\left(\mathrm{C}_{\mathrm{d}}\right)$ is reduced by $17.7 \%$ for the modified wagon with the realistic wagon on an average.
\end{abstract}

\section{Introduction}

In rapid evolution, transportation is a key element. In general transport means either public transportation or goods transportation. Considering apart from the other transportation vehicles, Rail transport is the streamlined transportation in India. The primary types of Indian railways rolling stock are freight wagons, passenger coaches, and diesel, electric locomotives. In national transporter, the freight transport is the main segment of trading and development of infrastructure, fuel consumption is important for the country. Rail freight wagons are used for transmit of cargo and also these are unpowered vehicles. Indian freight railways transported nearly 1160 million tonne of freight tin a year. By overcoming the logistics limits the operating speed of the train increases and a way to enhance the rail transportation insistence. National transporter earns $60 \%$ of the revenue lead the way on freight trains in India. Indian Railways are taken steps to increase the mean speed from the several perspective of the last decade, so the intention is to increase the fuel efficiency in a drastic manner to understanding the aerodynamic prospective \& aerodynamic changes. Now a days, many researchers concentrating on CFD simulation at different areas like IC Engines, aerodynamics [1]. Researchers examine about regional trains, high speed trains and also focusing on the aerodynamic approaches of freight trains. As per the studies of the researcher analyses the flow of turbulence behaviour of air by using the computational fluid dynamics and also effect of drag compared at the two configurations especially with and without vertical stiffeners on the outer walls and also to compare their effect on the aerodynamic coefficient. The result depicts the parameters of aerodynamic and simulated the stagnant position at zero yaw angles [2]. It was introduced the numerical analysis of unsteady nature of flow is complex around simplified tractor - aerodynamic performance of a regional passenger train for crosswind stability. The results represent the nature of flow characteristics around the train effected at different yaw angles [3]. This was simulated the steady flow for different rear slant angles and the result depicts the values of drag and lift coefficient are randomly dispersed and the flow separation occurred and the flow becomes unattached at different slant angles and also at rear slant angles. Pressure distribution is more equalized between bottom and top surfaces. The wake region clearly showed by the velocity contours, and continuously drag decreased as the rear angle is increased for the vehicle [4].It was presented the experimental results and compared with the air flow over ground vehicle is analysed and used as test case. The results found that the drag force for ground clearance and radius are different and simulated the body to get drag forces at various dimensions and positions to found that the optimum condition at which coefficient of drag is minimum. When increase and decrease the radius, the side edge of slant there is strong longitudinal vortices are formed and also when increase and decreases the ground clearance the nature of flow is fully separated and without vortices formed. For ground clearance the wake region increased in the vertical direction as compared with front radius[5].It was observed that the flow characteristics over a car model. The result depicts the parameters of aerodynamic coefficient and also compared them with available experimental data from this the numerical results are conceded [6]. It was analyses that the unsteady flow of the trains, passenger cars and around heavy vehicles. In this geometry mainly focused on the gap between the four variant vehicles. Large eddy simulations are used for simulated the flow around the four variant models. The result depicts the 
trailer and the flow is control for the passenger car and the flow is explored around the container freight. For regional trains PAN equations were used to simulate the flow but it is not related for the experimental data [6]. It was studied that the effects of various types of ground simulations on wind tunnel measurements of the drag coefficients and effects of Reynolds number considered [8]. This was simulated with two cases around the container freight wagon with one single wagon and submerged wagon. The result depicts the nature of flow around the single wagon is varied and expected to the flow around the wagon founded [9].Researcher presented detailed analysis of ride quality and rail aerodynamics evaluation methods. In this paper two and three dimensional theories for wheel-to-track interaction performance characteristics such as lateral stability, curving, multi body simulation, wheel to track interaction, ride quality and comfort are discussed. In this study the effect of roller radius, cone angle and wheel set profile on hunting speed was examined [1013]. This wascompared the behaviour analyses of cross wind for high speed train and low speed train. The results illustrate the cross wind curves of two trains for different values of the uncompensated lateral acceleration and also the function of the wind speed and train speed [14]. It was examined about the numerical simulation of a regional train in cross stability. The results represent the force coefficients, moment, DDESSA approach. For the reasonable level of accuracy, RANS methodology be used for the forecast of load coefficients and also simulated for the blunt train \& yaw angles as used by this methodology [15]. It was found that the performance of aerodynamic at different lengths of high speed trains without and with wind breaks, under a cross wind aerodynamic coefficients are carried out at different lengths were simulated [16].

\section{Aerodynamic theory}

Aerodynamics is the study of external flow around the bodies. It helps to determine the flow conditions and also improve the performance. In almost all the transportation devices, aerodynamic plays a crucial role and the structure of the vehicles exposed by the external flows it is the most prominent analysis of the flow arises the velocity and pressure distribution. The flow behavior was examined by the Continuity equation and Navierstokes equation.

Drag coefficient: The aerodynamic coefficient of drag is considering the effectiveness of the stream line body shape in reducing the air resistance to the forward motion of the vehicle.

Governing Equations:

To simulate the incompressible flow, Navier-Stokes equations for this flow type will be solved. The form of these equations based on the assumption follows:
Continuity equation,

$$
\frac{\partial \mathrm{p}}{\partial t}+\frac{\partial(\rho \mathrm{u})}{\partial x}+\frac{\partial(\rho \mathrm{v})}{\partial y}+\frac{\partial(\rho \mathrm{w})}{\partial z}=0
$$

For an incompressible flow,

$$
\frac{\partial \mathrm{p}}{\partial t}=0, \quad \nabla \cdot(\rho \bar{v})=0
$$

Thus;

$$
\frac{\partial \mathrm{u}}{\partial x}+\frac{\partial \mathrm{v}}{\partial y}+\frac{\partial \mathrm{w}}{\partial z}=0
$$

Where,

$$
\rho=C, \quad \nabla \cdot \bar{v}=0
$$

\section{Modeling and Boundary conditions}

The current wagon is variant of BOXN type and some standard features of BOXNHS wagon [10] are listed in Table 1.

Table 1: Standard features of BOXNHS wagon

\begin{tabular}{|c|c|c|}
\hline S.no. & Particulars & Parameter \\
\hline 1 & Length over headstock & $9784 \mathrm{~mm}$ \\
\hline 2 & $\begin{array}{c}\text { Length over } \\
\text { buffer/couplers }\end{array}$ & $10713 \mathrm{~mm}$ \\
\hline 3 & Length inside & $9784 \mathrm{~mm}$ \\
\hline 4 & Width inside/overall & $\begin{array}{c}2950 / 3200 \\
\mathrm{~mm}\end{array}$ \\
\hline 5 & Height inside/from rail & $\begin{array}{c}1950 / 3233 \\
\mathrm{~mm}\end{array}$ \\
\hline 6 & Bogie centres & $6524 \mathrm{~mm}$ \\
\hline 7 & Journal centres & $2260 \mathrm{~mm}$ \\
\hline 8 & $\begin{array}{c}\text { Wheel diameter on } \\
\text { tread }\end{array}$ & $1000 \mathrm{~mm}$ \\
\hline 9 & $\begin{array}{c}\text { Nominal maximum } \\
\text { axle load }\end{array}$ & $20.32 \mathrm{t}$ \\
\hline 10 & Tare & $23.1 \mathrm{t}$ \\
\hline 11 & Payload & $58.18 \mathrm{t}$ \\
\hline 12 & Ratio payload/tare & 2.52 \\
\hline 13 & Gross load & $81.28 \mathrm{t}$ \\
\hline 14 & $\begin{array}{c}\text { Number of wagons per } \\
\text { rake }\end{array}$ & 58 \\
\hline 15 & Throughput per rake & $3374 \mathrm{t}$ \\
\hline 16 & Loading density & $7.59 \mathrm{t} / \mathrm{m}$ \\
\hline 17 & Cubic capacity & $56.29 \mathrm{~m}{ }^{3}$ \\
\hline 18 & Speed (empty/loaded) & $100 / 100$ \\
\hline
\end{tabular}

The current wagon of CAD model is developed by using CATIA. The two geometric models are developed,

Case1: A detailed real case model of single wagon type 'BOXNHS' is modeled using CATIA, correct tostandard dimensions as per Indian railways shown in fig: 1 . 
Case2: The model is modified with flexible filler plates that appended on the face of the wagon at a distance of $284 \mathrm{~mm}$ from the both ends to reduce the aerodynamic effects as shown in fig: 2 .

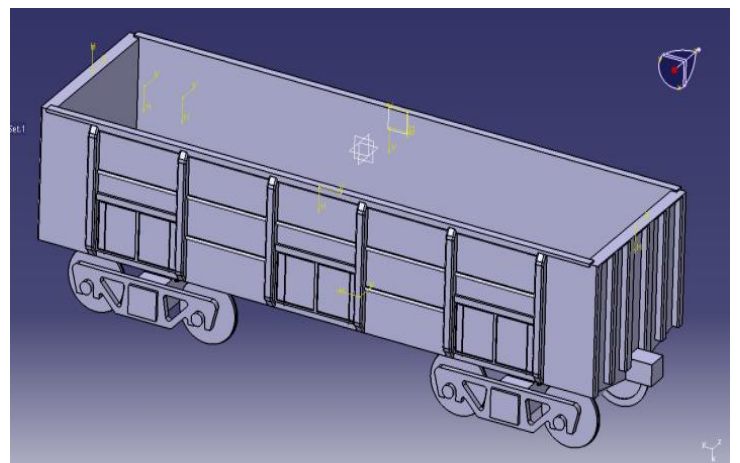

Fig.1: Real case single wagon geometry

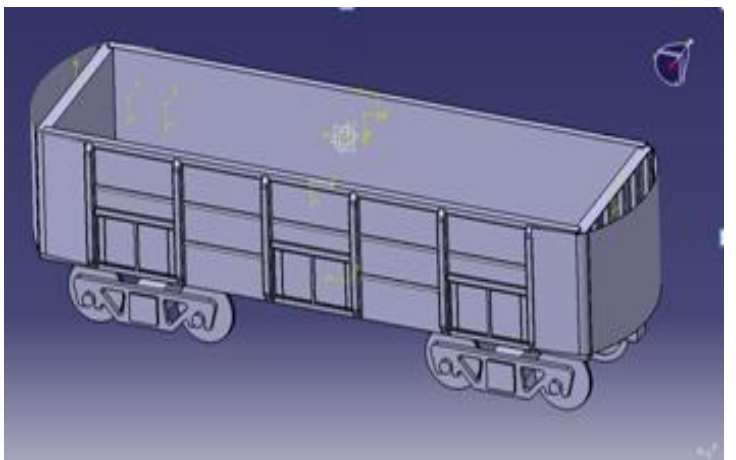

Fig. 2: Modified case single wagon geometry

The fillets are maintained as in original to get in close tolerance to the existing wagon in real time. By using the fluid flow analysis, the simulation of freight wagon subjected at zero yaw angles with ANSYS FLUENT 18.1 was used. The two cases are compared that's shown in the fig: 1 and fig: 2 . Once the geometry models of freight wagon are developed, enclosure of the computational rectangular domain has been created. The mesh controls for the current wagon are sizing. The parameters of the mesh size function are proximity and curvature and the mesh relevance center, smoothing and also span angle center should be medium and the transition of mesh condition is slow. Selecting the governing equations to be solved i.e., realizable kepsilon with non-equilibrium equation used for the turbulence model and also select the materials used in the analysis; the air is the domain for this analysis. The various inlet velocities are $27.78,33.33,38.89 ; 44.44$ $\mathrm{m} / \mathrm{s}$ are given to the boundary conditions at zero yaw angles. The boundary conditions in following manner are:

a) For the surface of the freight wagon, no-slip standard analytical flow of the wall surfaces is utilized.

b) Based on this analysis, the outlet pressure is fixed to zero for this boundary condition

c) Specification of turbulence intensity ratio is $5 \%$ and turbulence viscosity ratio is 10 .
3.1Meshing - Simulation for the standard and the modified wagons are carried out using ANSYS Fluent 18.1.

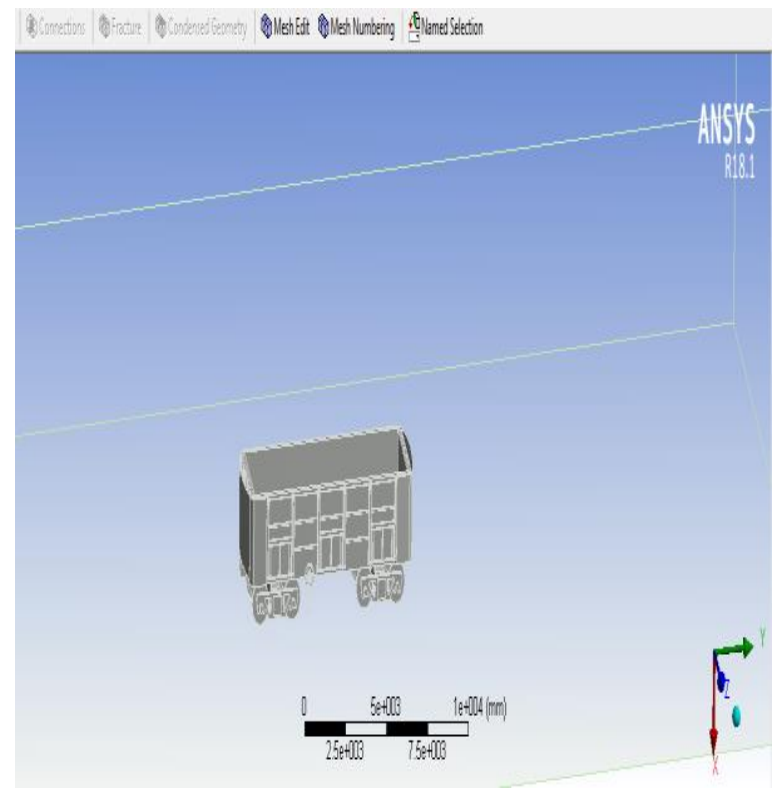

Fig. 3: Before Mesh for modified wagon

Mesh has been generated using tetrahedron element. The number of elements taken for meshing was 12104062 and nodes 2341267 in which the accuracy is high and the mesh cross section is shown in the fig: 4

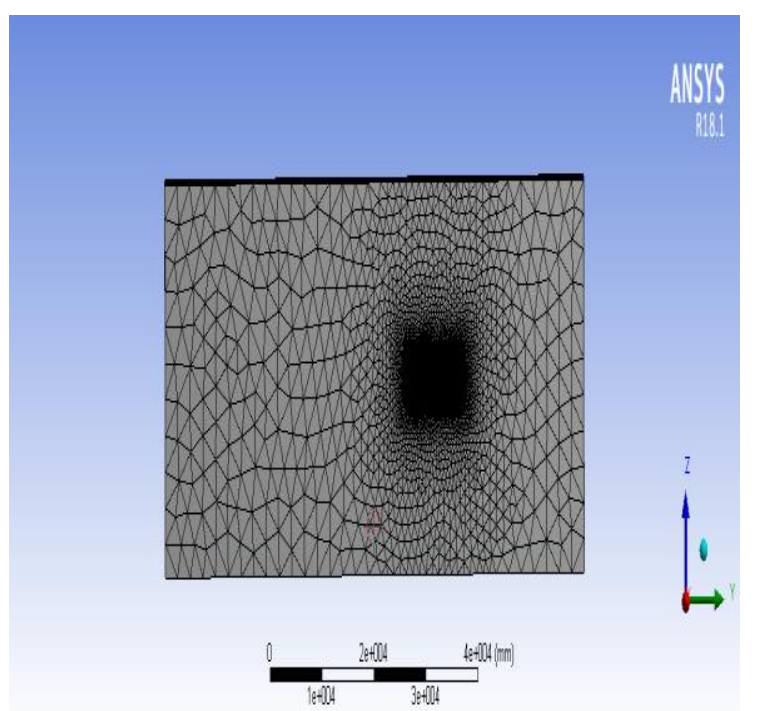

Fig. 4: After Mesh for modified wagon 


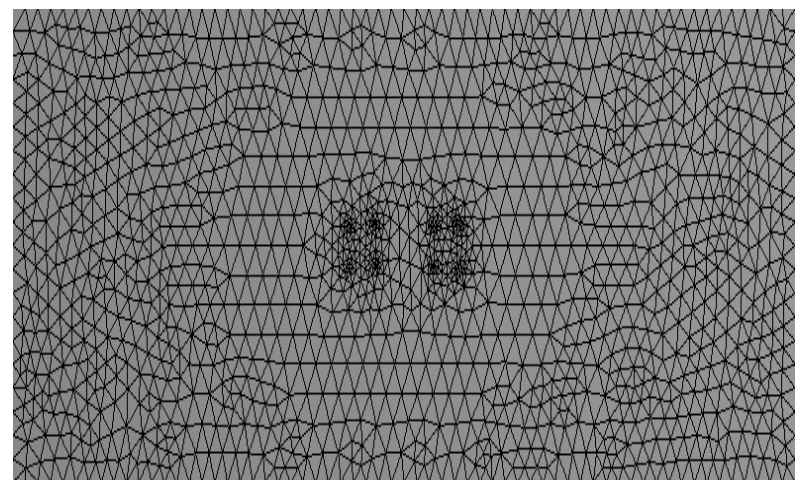

Fig. 5: Tetrahedral mesh element

The distance between train geometry and velocity inlet is kept in such a way that it ensures the incoming flow to be with uniform velocity and pressure fields by the time it reaches the train. The computational domain is designed sufficiently large to reduce the near wall effects. Symmetric boundary conditions were used at the side and top walls of the computational domain considering the fact that zero shear slip wall occurs in viscous flow. Due to the large application in the automotive industry, the standard $\mathrm{k}-\varepsilon$ (2-equation) turbulence model with non-equilibrium wall treatment was used for turbulence modeling. The analysis is done for both types of cases and found that drag-coefficient is decreasing upon appending filler plates.

\section{Results and Discussion}

The nature of air flow over the realistic and modified wagon is studied in this paper. There is a decrease in coefficient of drag when compared, realistic wagon with modified wagon. It was found that the coefficient of drag $\left(C_{d}\right)$ is reduced by $17.7 \%$ for the modified wagon with the realistic wagon on an average. The possible reason for reduction in coefficient of drag is due to the filler plates placed between wagons. The wake region between the wagons is reduced by the filler plates placed.

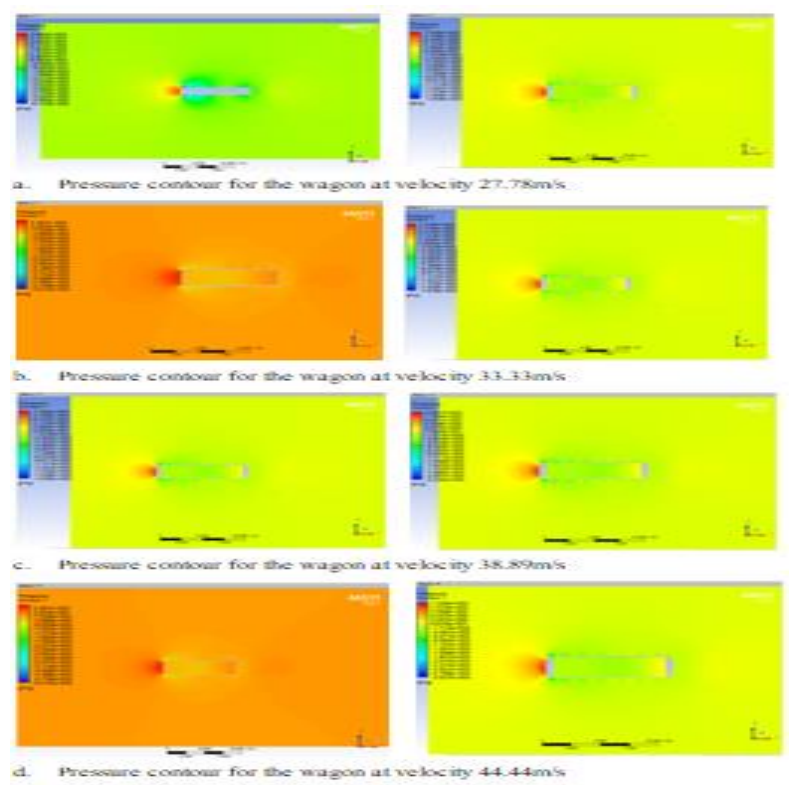

Fig.6: Pressure contour of wagon before and after modification
Fig. 6 shows the pressure contour for real wagon is more compared to the modified wagon because the face of appended filler plate at the front $\&$ rear end with modifications made to its geometry. This allows the flow to pass through the outlet without affecting the upstream flow.
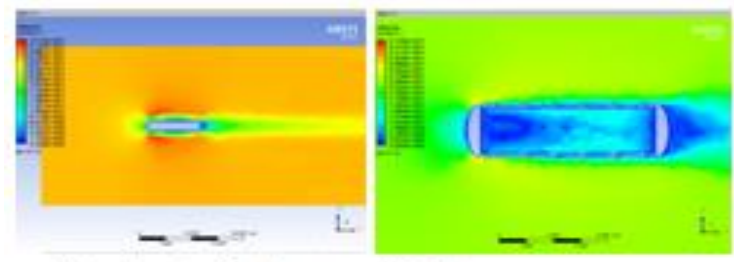

a. Velocity conow for the wagon at $27.78 m$ s
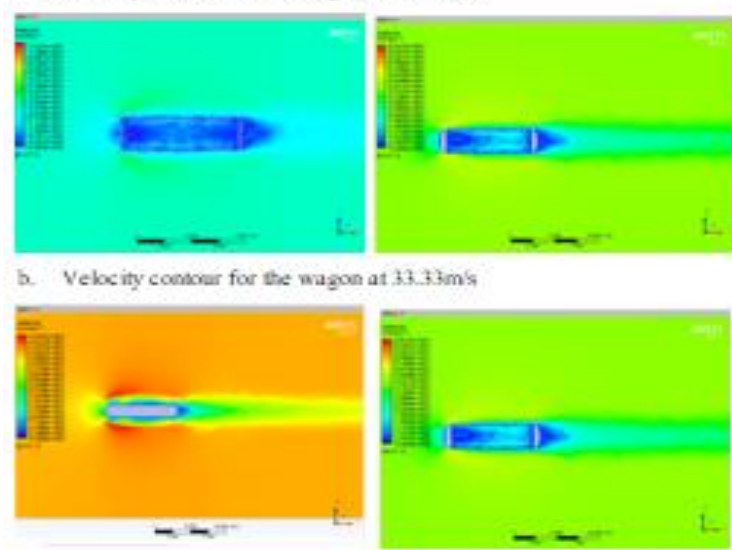

c. Velocity comoner for the wagon at $38.89 \mathrm{~m}$ s

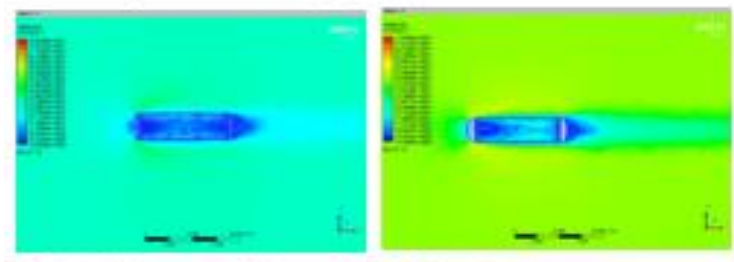

d. Velocity comber for the wagon at $44.44 \mathrm{~ms}$

Fig.7: Velocity contour of wagon before and after modification

Fig: 7 show the velocity contour for real and modified wagon varies between 27.78 to $44.44 \mathrm{~m} / \mathrm{s}$. The coefficient of drag slightly increases due to increase in velocity of real wagon due to the turbulence because of non-aerodynamic shape that is at inlet, the coefficient of drag decreases due to aerodynamic modification that is being modified at the inlet of the wagon so the flow passes over the body causing reduced or less turbulence.

It is validated with reference of "unsteady flow characteristics of the flow around the vehicle" (Ahmed) [6]. It is modeled as per standard experimental data in Catia and simulated using Ansys 18.1 with conditions that well suit our scenario and compared with the experimental data. The data is very close to the experimental data.

The validation fig. 7 shows the velocity correlated to the green area at $44.44 \mathrm{~m} / \mathrm{s}$ and the blue area show the low velocity and high pressure region. The wake region clearly indicates in the velocity contour. In consequence the drag formation is reduced as the wake area decreases and the flow over the modified wagon that is less 
turbulent. The wake region is less compared to real wagon in modified wagon. The appended filler plates allowed the air to flow over it with less turbulence there by reducing the drag.

The results of the pressure contour shows the proportionality of drag coefficient and the pressure is equalized the pressure was found to be reduced because the portion of the appended filler plate with the modifications made to its geometry, this is in turn to reduce the tendency of formation of a wake.

The results of the velocity contour shows that drag coefficient is proportional to the velocity because the velocity contour indicates the wake region and consequently drag formation decreases

The graph plotted to coefficient of drag for both standard and modified cases is presented.

Graph 1: Coefficient of drag for real wagon and modified wagon

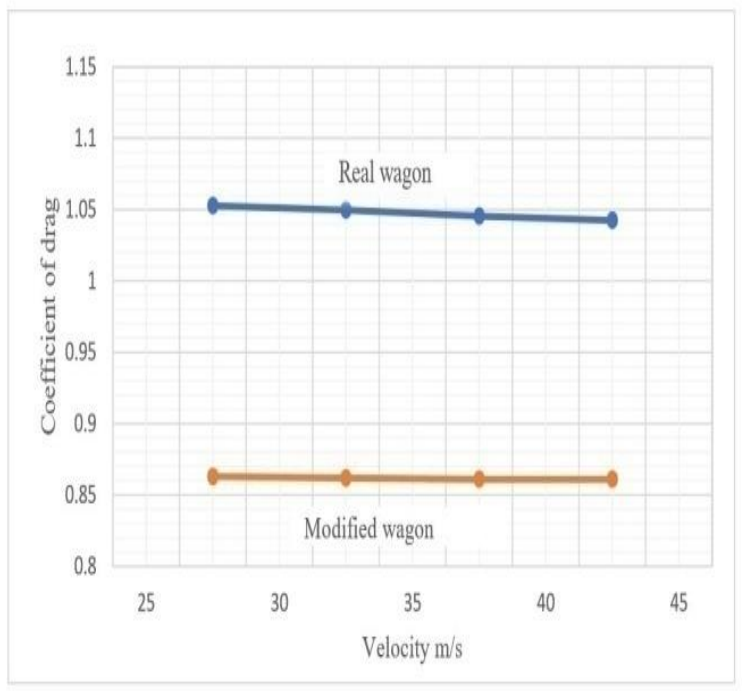

Graph1 shows the result of real wagon and modified wagon the drag has been reduced by $17.7 \%$ by appending filler plate to the real time wagon, this the great reduction in drag coefficient which is in reduced drag force because coefficient of drag is directly proportional to drag force.

\section{CONCLUSIONS}

Various factors such as coefficient of drag and velocity $\&$ pressure profiles are studied on BOXNHS wagon and compared the values of the modified model with that of the model of standard dimensions. It has been observed that the coefficient of drag is reduced which leads to an increase in aerodynamic efficiency. The wake region is nullified by appending filler plates in between the wagons. The fuel efficiency can be increased by taking this modification into effect.
Nomenclature

\begin{tabular}{|c|l|}
\hline BOXN-HS & $\begin{array}{l}\text { B-Bogie, O-Open, X-High sided, } \\
\text { N-Pneumatic/Airbrake, HS- High } \\
\text { Speed }\end{array}$ \\
\hline $\mathrm{F}_{\mathrm{d}}$ & Drag force \\
\hline $\mathrm{C}_{\mathrm{d}}$ & coefficient of drag \\
\hline $\mathrm{K}$ & Turbulence kinetic energy \\
\hline $\mathrm{E}$ & Turbulence dissipation rate \\
\hline
\end{tabular}

\section{References}

1. U.S. Jyothi \& K. Vijaya kumar Reddy "CFD analysis on particle diameter in hydrogen-enriched diesel engine for different piston bowl geometries", InternationalJournal of Ambient Energy, DOI:10.1080/01430750.2019.1710565. (2020)

2. Shashank B. Kedare, S.C. Sharma and S.P. Harsha"Computational Fluid Dynamics Analysis of Empty Railway Freight Wagons", in International Journal of Vehicle Structures \& Systems, 7(1), pp: 25-30.(2015).

3. M. Mohebbi and M.A. Rezvani"Numerical Analysis of Aerodynamic Performance of Regional PassengerAerodynamicsSAETechnicalPaperdoi:10. 4271/2001-01-2742.(2001)

4. Train under Crosswind Conditions" Int. J. Vehicle

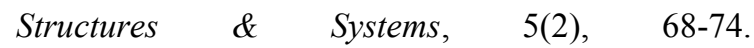
doi:10.4273/ijvss.5.2.05 (2013).

5. I.Bayraktar, D. Landman, and O. Baysal "Experimental and Computational Investigation of Ahmed Body for Ground Vehicle.

6. Ahmed, S.R ,Ramm, G.Faitin, Some Salient Features of the Time -" Averaged Ground Vehicle Wake", Society of Automotive Engineers (1984)

7. SaurabhBanga, Md. Zunaid, Naushad Ahmad Ansari,SagarSharma,RohitSinghDungriyal"CFDSim ulationofFlowaroundExternalVehicle:AhmedBod",I OSRJournalofMechanicalandCivilEngineering,:doi: 10.9790/168412438794.(2015)“

8. J.Osth"Unsteady Numerical Simulations of Flow around Heavy Vehicles, Trains and Passenger Cars"Thesis for the degree of Licentiate of Engineering inThermo and fluid dynamics (2012)

9. Baker, N.J. Brockie,"The aerodynamic drag of high speed trains,"Journal of Wind Engineering and Industrial Aerodynamics,PhD Thesis, University of Not- tingham, U.K.,(1988)

10. Yogeswara Reddy B, Srinivas Rao J, Suresh Kumar $\mathrm{T}$, Nagarjuna A, International Journal of Innovative Technology and Exploring Engineering, 8(11), 2019, 1194-1198

11. R.C. Sharma. "Stability and Eigenvalue analysis of an Indian Railway general sleeper coach using Lagrangian dynamics", Int. J. Vehicle Structures \& Systems, 5(1),9-14.(2013) http://dx.doi.org/10.4273/ijvss.5.1.02.

12. R.C. Sharma. "Recent advances in railway vehicle dynamics", Int. J. Vehicle Structures \& 
Systems,4(2),52-63.(2012)

http://dx.doi.org/10.4273/ijvss.4.2.04.

13. R.C. Sharma. "Parametric analysis of rail vehicle parameters influencing ride behaviour,"Int. J. Engg. Sci.\& Tech, 3(8), 54-65.(2011)

14. R.C. Sharma "Ride analysis of an Indian Railway coach using Lagrangian dynamics", Int. J. Vehicle Structures\&Systems,3(4),219-224.

http://dx.doi.org/10.4273/ijvss.3.4.02.(2011)

15. Zanetti "cross wind behavior on a high speed train and on a modern time $\mathrm{u}$ for urban and suburban transportation", j.wind engineering (2016)

16. Michael, Frank "Numerical simulation of a regional train in a cross wind journal of rail and rapid transit" DOI:10.1177/0954409714555383.(2014)

17. JiqiangNiu, Dan Zhou \&Xifeng Liang "Numerical investigation of the aerodynamic characteristics of high-speed trains of different lengths under crosswindwithorwithoutwindbreaks, EngineeringApp licationsofComputationalFluidMechanics, 12:1,195215,DOI:10.1080/19942060.2017.1390786C.J.

(2018) 